\title{
Migration, urbanisation et émergence des transgenres wallisiennes dans la ville de Nouméa
}

Migration, urbanization and increasing of Wallisian transgenders in Nouméa city

\section{Maroua Marmouch}

\section{OpenEdition}

Journals

Édition électronique

URL : http://journals.openedition.org/jso/7822

DOI : $10.4000 /$ jso.7822

ISSN : $1760-7256$

Éditeur

Société des océanistes

Édition imprimée

Date de publication : 15 décembre 2017

Pagination : 185-194

ISSN : 0300-953x

Référence électronique

Maroua Marmouch, « Migration, urbanisation et émergence des transgenres wallisiennes dans la ville de Nouméa », Journal de la Société des Océanistes [En ligne], 144-145 | 2017, mis en ligne le 15

décembre 2019, consulté le 15 mars 2021. URL : http://journals.openedition.org/jso/7822 ; DOI : https://doi.org/10.4000/jso.7822

\section{cc) (†)}

Journal de la société des océanistes est mis à disposition selon les termes de la Licence Creative Commons Attribution - Pas d'Utilisation Commerciale - Pas de Modification 4.0 International. 


\section{Migration, urbanisation et émergence des trans- genres wallisiennes dans la ville de Nouméa}

par

Maroua MARMOUCH*

\section{RÉSUMÉ}

Depuis quelques décennies, à Nouméa, chef-lieu de la collectivité d'outre-mer française de Nouvelle-Calédonie, les transgenres (hommes pour femmes) d'origine wallisienne ont acquis une visibilité croissante. La plupart d'entre elles affichent une performance de genre permanente, à travers l'habillement et le maquillage, et sont réputées s'adonner à la prostitution (tai'ata). Dans l'île d'origine, Wallis-etFutuna, leurs consours fakafafine (les transgenres locales) restent en revanche discrètes dans leur performance de genre et le tai'ata y est inconnu. Il s'agit dans cet article de décrire et de comprendre dans une perspective historique le phénomène des transgenres wallisiennes à Nouméa, et son rapport aux mutations urbaines, afin d'expliquer les raisons de leur visibilité croissante. Cet article suggère qu'elle est liée aux transformations démographiques, sociales et économiques survenues à partir des années 1970 dans la ville de Nouméa.

MoTS-CLÉS : transgenre, prostitution, urbanisation, Nouméa, Nouvelle-Calédonie, immigration, Walliset-Futuna

Aujourd'hui, à Nouméa, chef-lieu de la Nouvelle-Calédonie (collectivité d'outremer française), on croise régulièrement des personnes nées biologiquement hommes qui s'identifient comme appartenant au genre féminin. Les pratiques et les catégories que nous cherchons à comprendre ici, désignées comme "efféminées ", "travesties " ou " trans », seront appelées « transgenres », bien que

\begin{abstract}
Over the last decades, transgenders (Male to Female) of Wallisian origins have gained increasing visibility in Nouméa, New Caledonia. By now, most of them have adopted a permanent gender performance, which they express through dressing style and make up. They are widely associated with street sex work (tai'ata). Back in their place of origin, Wallis and Futuna, local transgenders, the fakafafine, are keeping a low profile gender performance and taiata is unknown. In this paper, we will describe the phenomenon of Wallisian transgender in Nouméa, and their relation to urban transformations, in order to understand the reasons of their increasing visibility. This paper suggests that their visibility needs to be understood in relation to demographic, social and economical transformations which affected Nouméa since the 1970s.
\end{abstract}

KeYwords: Transgender, Prostitution, Urbanisation, Nouméa, New-Caledonia, Immigration, Wallis and Futuna ce soit un terme débattu, de la même manière que " homosexuel », " gay », " lesbienne ». Ce terme désigne l'ensemble des personnes nées de sexe masculin mais qui se définissent comme étant de genre féminin et parlent d'elles-mêmes au féminin, qu'elles aient ou non changé physiquement de sexe ${ }^{1}$.

La plupart de ces transgenres sont issues de familles immigrantes d'origine wallisienne ou futunienne ${ }^{2}$,

1. Elles sont aussi appelées en anglais Male to Female ou MTF.

2. Par la suite, on dira «wallisienne " en suivant l'usage local où ce terme désigne les deux communautés originaires des îles de Wallis-et-Futuna, par volonté d'abréger l'appellation de «Wallisiens et Futuniens » et justifié par le fait que la plupart

* Docteur en Anthropologie sociale, EHESs-CREDO, 2015, marmouch.maroua@gmail.com 
arrivées en Nouvelle-Calédonie depuis les années 1950-1960. La plupart d'entre elles vivent quotidiennement sous une apparence féminine (jupes ou robes, maquillage, talons aiguilles...); d'autres affichent une féminité plus discrète (des hauts moulants avec des pantalons serrés) dans la journée et en famille, adoptant en revanche une apparence féminine en compagnie des " copines » et en soirée. Elles s'appellent en général par leurs nouveaux prénoms féminins d'adoption, dont certains sont aussi courants parmi les jeunes femmes de la communauté (Paula, Pamela, Sonia, Madone, Lola...), ou bien en employant l'expression «ma sœur ». En NouvelleCalédonie, on les désigne par des dénominations péjoratives d'origine française comme "pépètes ", "petalé» (qui vient de "pédale »), "efféminées". À Nouméa, beaucoup parmi les transgenres wallisiennes s'impliquent, pour des périodes plus ou moins longues, dans le monde de la prostitution de rue (tai'ata $\left.{ }^{3}\right)$. D'autres (en proportion mineure) ont des emplois variés et plus ou moins précaires, dans le domaine de la restauration, des transports en commun, dans la fonction publique, etc. On peut rencontrer également, en nombre plus limité, des transgenres d'origine tahitienne et/ou $\mathrm{kanak}^{4}$. La présence de ces dernières est devenue visible récemment, malgré la revendication répandue, dans les communautés kanak, de l'absence du "phénomène " transgenre : "chez nous, cela n'existe pas ". Dans cet article, en raison des contrainte de longueur et de choix méthodologique, il n'y aura pas d'analyse comparative entre la visibilité des transgenres wallisiennes et kanak (pour une analyse de l'émergence des transgenres kanak en Nouvelle-Calédonie, voir Marmouch, 2015).

Contrairement au cas des transgenres tahitiennes, connues sous le nom de $m \bar{a} h \bar{u}^{5}$, et dont la présence est attestée historiquement (Besnier, 1994), l'existence des "hommes comme les femmes " wallisiennes (fakafafine) à l'époque du premier contact occidental avec la société wallisienne n'est pas mentionnée dans les comptes rendus des missionnaires maristes, dont les premiers datent de 1838, ni dans les rapports d'administration du protectorat (1887-1961). Or, malgré le silence des références historiques et anthropologiques, les fakafafine ont probablement toujours existé à Wallis. En effet, selon les transgenres d'origine wallisienne rencontrées à Nouméa, il existe bien des fakafafine à Wallis, bien que leur nombre reste limité et leur "performance" transgenre discrète (elles ne portent pas quotidiennement de vêtements féminins, par exemple). À Nouméa, les transgenres sont de plus en plus visibles et, selon les témoignages recueillis, de façon récente. Elle peut être située dans la ville de Nouméa à partir de la moitié des années 1970. Comment comprendre l'écart de visibilité des transgenres wallisiennes dans l'île d'origine et dans l'immigration ? Ou, pour le dire autrement, pourquoi les transgenres wallisiennes affichent-elles une performance de genre apparente et s'adonnent-elles à la prostitution de rue (tai'ata) dans l'immigration, en Nouvelle-Calédonie, alors qu’à Wallis elles ne sont pas visibles et que le tai'ata reste inexistant?

La réponse à cette question doit être recherchée dans une perspective historique longue, et spécialement dans les changements socioéconomiques et urbains qu'a connus le territoire durant la période de l'installation des familles wallisiennes dès les années 1950-1960 à Nouméa. L'objectif est donc de comprendre ici le processus par lequel les transgenres d'origine wallisienne à Nouméa ont acquis une visibilité croissante à Nouméa, en analysant leur apparition, leur mode de vie et leur évolution actuelle en relation avec l'urbanisation de la ville.

À cet effet, cet article se base sur les récits de vie de neufs transgenres, recueillis durant mon séjour de terrain entre 2012 et $2014^{6}$. Dans un premier temps, j'analyserai le contexte historique de l'émergence des transgenres wallisiennes à Nouméa. Cela permettra, dans un second temps, de souligner l'impact que les mutations urbaines ont eu sur la performance des transgenres, leur conférant de plus en plus de visibilité. Finale-

de mes informatrices utilisent elles-mêmes cette dénomination englobante, quelle que soit l'île d'origine de ces personnes. Cette appellation n'est pratiquée qu'à Nouméa car les habitants des îles de Futuna tendent à se distinguer par rapport à ceux de l'île voisine, considérant que Wallis maintient une attitude "colonialiste" vis-à-vis de Futuna, en centralisant les services et l'argent.

3. Mot d'origine tahitienne défini par le dictionnaire tahitien-français de l'académie tahitienne comme "débauche, luxure, impudicité".

4. Durant mon séjour en Nouvelle-Calédonie, je n’ai rencontré qu’une seule transgenre "blanche ». Par ailleurs, mes enquêtées m’ont confirmé l'absence de personnes transgenres d'origine européenne. Cela mérite d'être étudié et analysé dans une recherche ethnographique ultérieure.

5. $M \bar{a} h \bar{u}$, en tahitien, est communément utilisé pour des personnes nées garçons mais qui manifestent dès l'enfance des manières considérées comme étant féminines, telles qu'une inclination pour les travaux d'entretien de la maison ou les soins et les services à la famille et aux enfants. La féminité des māhū est manifestée à travers les manières et les activités, mais elle ne se manifeste jamais par l'habillement ou l'affichage des relations sexuelles avec les hommes. Les māhū semblent ainsi être acceptées dans le système des valeurs de la société et de la culture tahitienne. On les retrouve aujourd'hui autour d'activités de soins et de services, dans les services de restauration et de tourisme, ainsi que dans les arts et le spectacle.

6. L'enquête de terrain, effectuée en deux temps, de juillet 2012 à avril 2013 et d'octobre à décembre 2014, s'est concentrée principalement sur la région du Grand Nouméa, où j'ai pu m’entretenir avec neuf transgenres d'origine wallisienne et, dans une moindre mesure, dans la province Nord de la Grande Terre, où j'ai pu interviewer trois transgenres kanak. L'âge des transgenres rencontrées dans le cadre de cette recherche allait de 19 à 52 ans lors de notre première rencontre. Parmi les douze personnes interviewées, deux font partie de la première génération de transgenres. Les autres sont issues des générations suivantes. 
ment, je discuterai le rapport ambivalent des transgenres avec leurs familles, entre tolérance et marginalisation, ainsi que leur rapport avec leurs réseaux sociaux, en m'intéressant aux stratégies qu'elles développent aujourd'hui afin de résister à la stigmatisation.

\section{Espaces urbains et émergence des transgenres dans la ville}

Dans nombre d'îles polynésiennes, les transgenres, comme les fakaleiti (Tonga), les raerae (Tahiti) et les fáafafine (Samoa), sont devenues plus nombreuses et plus visibles dans les petits centres urbains qui ont commencé à voir le jour dès les années soixante: Nuku'alofa, Papeete et Apia, etc. (Besnier, 1997 ; Elliston, 2014 ; Dolgoy, 2000). L'émergence des transgenres wallisiennes en Nouvelle-Calédonie se situe elle aussi, selon les témoignages recueillis, à cette période. Afin de comprendre le contexte de la visibilité des transgenres wallisiennes à Nouméa, j'ai demandé à Lola et Chantal de me raconter comment elles ont vécu leur féminité durant leur jeunesse. Toutes les deux ont la cinquantaine passée. Lola et Chantal sont nées en Nouvelle-Calédonie de parents immigrés wallisiens arrivés entre les années 1950 et 1960 ; elles ainsi font partie de la première génération de transgenres d'origine wallisienne nées à Nouméa. Bien qu'issues d'une réalité sociale et économique similaire, Lola et Chantal ont vécu deux parcours différents dans le cheminement vers la féminité. La première affiche des manières féminines tout en gardant une apparence vestimentaire et corporelle masculine. La seconde, en revanche, affiche une féminité sophistiquée: corps sexuellement opéré, maquillage et habillement d'influence occidentale (par exemple robe courte avec des talons). Selon Lola et Chantal, durant leur jeunesse (les années 1970), les transgenres wallisiennes de Nouméa ne s'habillaient jamais comme les femmes, ni en public ni devant leur famille. La performance transgenre se résumait ainsi, pour la plupart d'entre elles, aux " manières ", à la démarche, à la façon de parler et au fait de privilégier la compagnie des femmes, comme l'explique Lola dans cet extrait :

«Avant on n'était pas... on était une génération beaucoup montrée du doigt, et comme c'était une chose qui n'était pas encore... c'était nouveau. C'était pas encore dans notre ethnie [wallisienne], c'est-à-dire, s'habiller en femme et machin [...]. On se cachait beaucoup, même pour les manières. Pour pouvoir sortir tranquillement, il fallait franchement ressembler à un mec. Il fallait pas faire les manières des pépètes. Il fallait parler comme un mec. Et moi, j'étais obligée de faire ainsi. [...] On en avait beaucoup qui avaient les manières d'une femme, on était efféminée mais tout en restant masculin quoi... Chantal, elle, c'était la seule avec l'autorisation de ses parents qui s'habillait en femme, qui venait en femme. C'était la seule. Parce que ses parents l'autorisaient. » (Lola)

Selon Lola, ce sont les «travesties " tahitiennes qui arrivaient en Nouvelle-Calédonie pour travailler qui ont commencé les premières à « $s^{\prime}$ habiller comme les femmes " dans les lieux publics de Nouméa au milieu des années 1970. Ceci aurait influencé par la suite, selon elle, la performance de genre des fakafafine de la ville, comme elle le souligne ici :

«[...] À Tahiti, là-bas, ils étaient déjà à cette époque en pleine évolution de prostitution. Il faut croire que c'est les Tahitiennes qui sont arrivées de Tahiti, des travesties qui sont venus... C'est l'influence des Polynésiennes de Tahiti... Il y en a eu trois qui sont arrivées au début des années 1970, maintenant elles sont vieilles... Ce sont elles qui s'habillaient en femme. " (Lola)

Ces « travesties » étaient vraisemblablement les premières raerae tahitiennes, qui ont fait leur apparition à partir des années soixante en Polynésie française ${ }^{7}$ (Elliston, 2014 ; Bauer, 2002). Les raerae - terme tahitien, popularisé dans tout le Pacifique francophone, et au-delà, par la littérature journalistique - sont les transgenres nées garçons et devenues filles. Figures surtout urbaines, très visibles, à l'habillement féminin (à l'occidentale) et glamour, les raerae sont souvent associées au monde de la prostitution.

Larrivée des raerae sur le Territoire s'inscrit dans une période d'afflux de migrants en NouvelleCalédonie, qui coïncide avec un processus d'urbanisation. En effet, à l'instar des centres urbains d'autres îles du Pacifique (Dussy et Wittersheim, 2013), Nouméa connut un véritable changement économique, social et démographique à la fin des années soixante et au début des années soixantedix. Le «boom du nickel» (1968-1972) déclencha une augmentation des activités économiques et une demande croissante de main d'œuvre. Pour faire face à la grande demande du marché du travail, et pour faire barrage aux Kanak dans l'accès à l'emploi après l'abolition du "statut de l'indigénat " (Guiart, 1996 : 244), l'État français encouragea une migration massive polynésienne vers la Nouvelle-Calédonie (Angleviel, 2006:

7. Les raerae sont les transgenres (hommes pour femmes) de Polynésie française. Cette figure transgenre est apparue dans la ville de Papeete durant les années 1960, période marquée par l'urbanisation et les changements économiques en Polynésie française. À l'instar des transgenres wallisiennes de Nouméa, les raerae manifestent au quotidien leur féminité à travers l'habillement féminin à la mode occidentale (robes, jupes...), le maquillage, la pratique de la prostitution et la prédilection pour la vie nocturne. Elles se différencient ainsi des mähü dont la performance de la féminité se borne aux manières et aux activités féminines. 
73 ; Pechberty, 2004 : 65). L'immigration polynésienne était suivie par l'arrivé de travailleurs depuis la métropole (Freyss, 1995). Selon l'économiste Freyss, entre 35000 et 40000 travailleurs métropolitains sont ainsi arrivés sur le Territoire entre 1969 et 1976, et environ 20000 s'y sont installés durablement (Freyss, 1995). La plupart de ces nouveaux arrivés s'installa à Nouméa.

Selon Lola, les premières transgenres tahitiennes arrivées à Nouméa auraient été aussi les premières à proposer des services sexuels en échange d'argent. Lola explique que les raerae tahitiennes rencontraient leurs potentiels « clients" dans les nouveaux lieux de divertissement qui ont commencé à voir le jour dans la ville, tels que les bars et les boîtes de nuit. Selon les témoignages recueillis, il apparaît que parmi les hommes métropolitains, arrivés en tant que fonctionnaires, militaires et ingénieurs, quelques-uns cherchaient à entretenir des relations sexuelles en échange d'argent - pratique rare sinon inexistante à cette époque en Nouvelle-Calédonie. Par la suite, quelques fakafafine locales, encouragées par la présence des raerae tahitiennes et par l'anonymat que ces lieux de rencontre assuraient, les auraient ainsi rejoints. C'est à cette période que, imitant les raerae, Lola et quelques autres jeunes fakafafine auraient ainsi commencé à adopter une performance féminine au moyen de vêtements, de maquillage et de talons, afin d attirer une clientèle d'hommes prêts à payer pour des services sexuels. Cependant, selon Lola, cette féminisation ainsi que les rencontres avec les hommes métropolitains ne se produisaient que dans ces lieux nocturnes, loin du contrôle familial, comme elle le souligne dans cet extrait :

« [...] Et puis voilà, quoi... À 16 ans, moi aussi j'ai commencé à tapiner en boîte, au centre-ville. Ils y avaient les militaires [...] Je m'habillais normalement en garçon, arrivée là-bas, je mettais une robe dans les toilettes et... voilà ! Je cachais mon linge dans un coin et voilà, je descendais en femme en boîte [...] Dans les années 1978-79, on n'était pas nombreuses. Voilà, les travesties commençaient à faire leur apparition et on commençait à se faire montrer du doigt. En toilettes, dans les coins, on était un peu partout dans les boîtes. La police tolérait [...] Moi, je faisais que la nuit, le matin j'étais en garçon. » (Lola)

Ainsi, c'est dans les lieux nocturnes que Lola et d'autres transgenres wallisiennes ont porté pour la première fois des habits féminins et attirants, devant lesquels les clients "blancs 8 " étaient " éblouis ", selon ses propres dires. Les nouveaux lieux urbains nocturnes auraient ainsi incarné le premier espace de la manifestation de la perfor- mance de la féminité transgenre en Nouvelle-Calédonie à travers les pratiques vestimentaires et la compagnie des hommes, durant les années 1970, loin des yeux désapprobateurs de la famille ou de la communauté comme l'explique ici Chantal :

«[...] Moi, quand j'ai commencé à aller, j'avais 17 ans [...] J'allais parce que, si tu veux, comme tu pouvais pas t'habiller comme femme ailleurs, il y avait que là qu'on se sentait femme. Parce que la plupart des mecs qui passaient te faisaient des compliments [...].»(Chantal)

\section{Nouvelles mutations urbaines et impact sur la « performance » transgenre}

Le «boom du nickel ", l'immigration et le processus d'urbanisation ont contribué à transformer les comportements sociaux des Wallisiens de Nouméa, ainsi que des autres " communautés " vivant dans la ville (voir à ce propos, sur les Kanak «de Nouméa ", Hamelin, 2000). Depuis les années 1970 s'est diffusée dans la ville une pluralisation des modes de vie, des conduites et des comportements (Guiart, 1996 ; Hamelin, 2000 : 344). On assiste ainsi, au cours des dernières décennies, au développement d'une classe moyenne urbaine wallisienne, où l'un des conjoints a un emploi aussi rémunérateur que ceux des "blancs", dans la fonction publique, en CDI, etc. Cette classe moyenne urbaine émergente développe de nouveaux besoins en termes d'alimentation (intérêt pour les nourritures achetées et consommées à l'extérieur), de loisirs (aller au cinéma, regarder la télé, danser en boîte de nuit...) et de consommation (voiture, voyages, nouveaux habits à l'occidentale). En effet, l'adoption de modes vestimentaires d'influence occidentale chez les jeunes des deux sexes s'est généralisée au cours des dernières décennies en Nouvelle-Calédonie, principalement à Nouméa. Des vêtements tels que les jeans et les t-shirts leur sont devenus habituels. Toutefois, les habits traditionnels wallisiens restent généralement portés lors des fêtes religieuses, des mariages et le dimanche à l'église. Néanmoins, on voit de plus en plus les femmes wallisiennes prendre soin quotidiennement de leur apparence vestimentaire et esthétique à la mode occidentale. Aussi, dans les lieux nocturnes, il n'est plus rare de voir de jeunes filles wallisiennes portant des habits "attirants" tels que des pantalons moulants et des robes courtes danser sur des tubes RnB américains. Rouge à lèvres, teinture de cheveux et talons sont devenus les symboles d'une féminité occidentale et moderne. En outre, le mode de vie

8. Durant ma recherche de terrain, je n'ai pas eu la possibilité d'analyser et de décrire les clients du taíata. Selon mes informatrices, ceux-ci sont issus de tous les groupes ethniques qui composent la Nouvelle-Calédonie (Caldoches, Wallisiens, Vietnamiens, Tahitiens et Kanak). Néanmoins, selon elles, les clients « blancs » représentent encore aujourd'hui la grande majorité. 


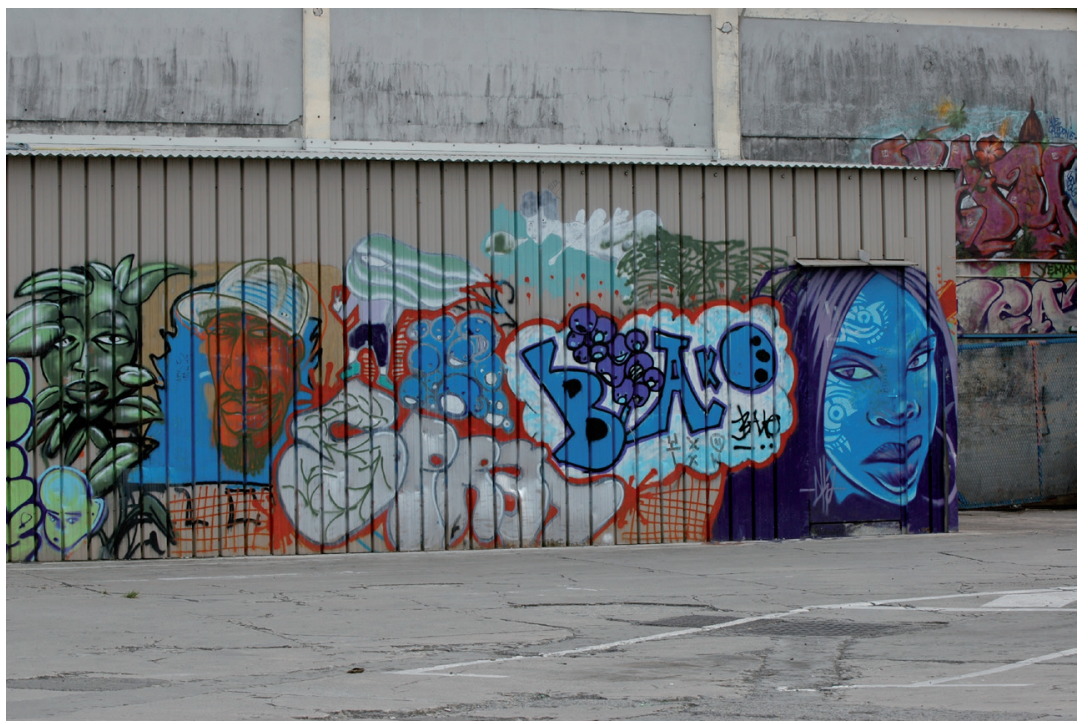

Рното 1. - Graffitis urbains, centre ville de Nouméa, 2011 (C) Isabelle Leblic) rapport à la famille et à la communauté d'origine (Pechberty, 2004 : 69). Ce processus peut également être observé à Wallis (Pechberty, 2004: 74), mais il est renforcé à Nouméa pour les raisons que j'ai exposées.

Ces mutations socioéconomiques ont eu un impact sur la conception de la corporéité et de la féminité des transgenres wallisiennes, ainsi que sur leurs modes de vie, au même titre que pour les autres catégories sociales. Aujourd'hui, même si la plupart d'entre elles des plus jeunes fait davantage de place à la mixité et l'école contribue à promouvoir plus de promiscuité entre les deux sexes (Salomon, 2000). Les enfants scolarisés des deux sexes sont poussés par leurs parents à poursuivre une ascension sociale. La généralisation des notions "d'amour romantique " et de libre choix amoureux, par la diffusion d'un modèle global à travers les mass-média, a favorisé pour sa part la diversification des pratiques matrimoniales, l'émergence de nouvelles expressions et de vécus plus libres des pratiques sexuelles (les mariages mixtes, le concubinage, les relations sexuelles avant le mariage...). La population wallisienne immigrée en Nouvelle-Calédonie maintient des liens avec la tradition de l'île de Wallis. En effet, on assiste toujours à des moments d'échange de dons et de contre-dons comme dans l'île d'origine durant les fêtes familiales de la communauté wallisienne à Nouméa (communions, baptêmes, mariages, etc.) (Pechberty, 2004 : 69 ; Leleivai, 2002 : 187). Par ailleurs, la plupart des Wallisiens de Nouvelle-Calédonie, jeunes et adultes, maintiennent une forte pratique religieuse, comme à Wallis-et-Futuna, notamment par une importante fréquentation des activités pastorales (construction de chapelles, bénévolat, formation, création de groupes de jeunes, etc.) (de Deckker, 1997 : 54). Néanmoins, il convient de souligner ici, qu'à côté du modèle français métropolitain déjà ancré, les changement intervenus au cours des dernières décennies principalement en ville, tels que l'influence croissante des médias (télévision, cinéma et récemment internet), les changements socioéconomiques, la judiciarisation et les programmes scolaires ont apporté des idéologies connexes telles que la "liberté personnelle " et la "réussite individuelle ». Autant de transformations qui sont à l'origine de la quête de mobilité sociale ascendante et de la recherche de l'autonomie individuelle par sont impliquées dans le monde de la prostitution de rue (tai'ata), de plus en plus nombreuses sont celles qui exercent, en parallèle du tai'ata, ou uniquement, des métiers salariés dans la restauration, en tant que fonctionnaire, chauffeur, etc. tout en affichant leur "féminité " cosmopolite à travers les pratiques vestimentaires et les soins esthétiques. Par ailleurs, pour les jeunes transgenres, les relations avec les hommes hétéronormatifs ne se résument plus aux rencontres du taiata mais sont de plus en plus orientées par le désir (rarement réalisé) de construire des relations affectives et sexuelles stables et déclarées avec des hommes, et principalement avec des hommes «blancs ". Ainsi, la nouvelle "performance" féminine transgenre ne se manifeste plus uniquement dans les espaces nocturnes, comme c'était le cas pour la génération de Lola. Aujourd'hui, on voit les transgenres porter au quotidien, en journée comme en soirée, dans la rue et dans les espaces publics (dans leur lieu de travail, les restaurants, la rue...) des habits féminins à l'européenne. Si les critères de la mode sont les mêmes pour les transgenres que pour les femmes de leur communauté, l'engouement pour une apparence corporelle "moderne", signifiée par les robes fashion et les soins esthétiques (épilation, coiffure moderne, cheveux peignés, maquillage soigné, piercing...), est généralement plutôt renforcé chez les transgenres. Elles inclinent à adhérer aux symboles de la beauté féminine cosmopolite telle qu'elle est véhiculée par les films, les clips et les magazines et à les importer localement (pour une comparaison avec le cas fakaleiti, voir Besnier, 1997, 2002 et 2013). Néanmoins, cette féminité de type "globale" suscite des réactions différentes et contradictoires de la part de leur famille et de la "communauté ", qui varient entre l'acceptation, voire l'admiration, mais souvent des situations de marginalisation et de rejet, car 
elle pourrait être jugé «immorale », comme on le verra par la suite.

\section{Rapport avec la famille et réseaux sociaux transgenres dans la ville}

Cette plus grande visibilité dans toutes les sphères de la vie et de la ville ne doit en effet pas laisser penser que les transgenres de Nouméa sont acceptées et tolérées sans friction. Les récits de vie recueillis témoignent ainsi de la difficulté à se faire accepter par la société dans son ensemble, et notamment par la "communauté » wallisienne, et reviennent souvent sur les situations de marginalisation, les insultes et la ridiculisation que les transgenres subissent. Bien qu'elles affichent, comme leurs homologues polynésiennes (Besnier, 1994 : 296), un efféminement par les manières et la gestuelle dès l'enfance, les transgenres wallisiennes déclarent en fait être élevées comme étant des garçons, et leurs parents attendent qu'elles adoptent un rôle d'homme. Dans leurs récits, ce sont principalement les pères et les frères qui sont décrits comme hostiles à l'efféminement, les poussant, dès leur enfance, à adopter des manières et des activités jugées masculines, à ne pas rester en compagnie des femmes, à couper leurs cheveux... Les mères et les grandes sœurs, en revanche, sont décrites comme plus tolérantes par rapport aux hommes de la famille, face à la visibilité de ces " aspects féminins ». Les critiques qui peuvent être adressées aux transgenres concernent principalement le comportement "extravagant" et l'adoption d'un mode de vie "léger ", où la recherche de relations sexuelles ou affectives avec les hommes est exprimée ouvertement. Déborah, par exemple, une transgenre de 26 ans, subit beaucoup de reproches de la part de sa famille. Comme beaucoup d'autres transgenres, elle affiche au quotidien une apparence féminine de type " occidentale " séduisante (robe, jupe courte, hauts moulants, maquillage...). Elle a subi une chirurgie de réassignation de sexe en Thaillande et a fréquenté pendant quelques années le monde de tai'ata. Durant un séjour de quelques semaines chez elle, dans la maison de ses parents, j’ai assisté plusieurs fois à des querelles entre elle et sa mère, cette dernière lui reprochant ses sorties nocturnes avec les autres "filles", sa recherche de relations avec des hommes, et son rapport à l'alcool (le fait qu'elle sente l'alcool en rentrant à la maison le lendemain au matin). Une des proches de Déborah, Leslie, explique ainsi les raisons du mécontentement de sa famille :

«[Déborah] est une femme, elle a [fait] le changement d'identité... Mais quand tu dis "femme", c'est un grand mot. Même le linge [l'habillement], ça montre le respect. Je veux dire, tu vois, quand on est en famille t'as des trucs [à respecter]. Je veux dire, tu t'habilles correctement. Quand tu vas aller en boîte, tu t'habilles sexy machin, mais durant la journée, en famille, au boulot, tu t'habilles correctement. [...] Déborah doit changer sa manière de se fringuer, surtout pour elle. Tout le monde sait qu'elle est une trans. Où on va, ils savent tout de suite que c'est une trans parce qu'elle fait tellement trop, que même avec sa voix... " (Leslie).

Comme on le voit dans cet extrait, les principales critiques envers les transgenres concernent leur habillement jugé "provocant " et «non respectueux ». La stigmatisation des transgenres comme " extravagantes ", et provocantes" par leurs manières et leurs vêtements glamours, empêche par ailleurs l'établissement de rapports amicaux avec les femmes hétéronormatives. Selon les témoignages recueillis, si les transgenres recherchent ces amitiés pour asseoir leur appartenance au monde féminin, certaines femmes hétéronormatives aiment également leurs compagnies et leurs "blagues" provocantes. Mais les tensions sont néanmoins fréquentes entres elles. D’une part, les transgenres se sentent toujours plus "belles" et plus «libres" que les « vraies" femmes, qui sont représentées dans leurs discours comme moins sophistiquées et plus conservatrices qu'elles, comme l'exprime Roby ici :

«Les filles $[\ldots]$ sont très pudiques. [...] Sont toujours là à complexer, à poser des questions. "

Leur beauté féminine "provocante " suscite par ailleurs, selon Amira, une transgenre de 26 ans, la jalousie des "vraies" femmes qui voient en elles des rivales, ce qui empêche très souvent l'installation d'une relation de confiance entres elles. La relation amicale avec les hommes de leur "communauté " n'est pas moins compliquée. Les rapports avec les hommes reconnus comme " hétérosexuels " restent superficiels, car ils acquièrent la plupart du temps un caractère sexuel ou d'attirance sexuelle (de la part de la transgenre ou de l'homme), ce qui ne permet pas la réalisation d'une relation seulement amicale.

La plupart des "filles" rencontrées affirment ainsi que les cercles amicaux composés essentiellement par les "copines " sont le milieu où elles se sentent le plus acceptées, celui dans lequel elles peuvent être elles-mêmes. Les " copines ", appelées aussi "sœurs", sont issues, pour la plupart, de la culture wallisienne. Les amitiés suivent donc une logique de voisinage communautaire, comme c'est le cas pour tous les autres membres de la société, femmes ou hommes. Se connaissant et se côtoyant dès l'enfance par le voisinage, le collège ou bien le même groupe de jeunes à l'église, la socialisation entre transgenres commence très tôt. Les "sœurs" partagent des étapes importantes de leur vie et se rencontrent plusieurs fois par semaine, souvent tous les jours. C'est en com- 
pagnie des "copines" qu'elles racontent avoir commencé à parler de leur attirance envers les garçons, à discuter de leur envie de féminisation, à se questionner sur les possibilités de la transition sexuelle. Parfois, les «sœurs » font partie aussi de la famille élargie en tant que cousines. Certaines " copines " sont appelées " mères" ou " grandessœurs » par les transgenres plus jeunes car celles-ci jouent le rôle de "marraines » ou de modèle dans la socialisation des novices. Dans les récits de vie recueillis, une "mère " ou une "grande-sœur" est décrite comme une transgenre de quelques années plus âgée, qui accompagne les différents moments du parcours de sa plus jeune "fille» : du choix du prénom féminin d'adoption, au style d'habillement, en passant par l'intégration dans les lieux de socialisation urbaine (discothèque, restaurants, bar) et, le plus souvent, jusqu'à l'introduction dans le monde du tai'ata. Dans le cas d'une rupture familiale suite à la féminisation de la jeune transgenre, c'est auprès d'une "grandesœur »ou d'une « mère » qui l'aide matériellement ou en l'hébergeant quelques temps chez elle que la jeune trouve refuge et solidarité. Ce sont aussi les «sœurs" qui parlent pour la première fois aux plus jeunes des possibilités de la transition chirurgicale et qui leur " passent l'info " - le plus souvent issue de leur propre expérience de transition chirurgicale en Thaillande -, leur conseillant la plupart du temps de se féminiser plus et d'être plus sexy. Les "sœurs" sont ainsi une source d'informations, de solidarité et d'affection qui leur permet de résister à la stigmatisation qui provoque la plupart du temps leur marginalisation des autres cercles de solidarité traditionnelle, comme les amitiés féminines ou les liens familiaux. On les voit ainsi toujours se déplacer en petits groupes dans les rues, au café, en boîte, chez l'une d'entre elles. Dans leurs récits, elles disent que ces amitiés leurs permettent de se défendre contre des insultes qui pourraient être proférées par des " mecs » dans la rue ou en boîte. Ces amitiés et la "filiation" de groupe sont néanmoins fragiles. Elles débouchent le plus souvent sur des rivalités qui peuvent provoquer des bagarres dans des lieux publics et des règlements de comptes, lorsqu'elles sont remises en question suite à des commérages, autour d'histoires d'hommes ou de rivalité (pour une analyse comparative des rapports ambigus et fragiles entre les " copines " travesties au Brésil, voir Kulick, 1998 : 40-42).

\section{Recherche de la «féminité " moderne et résistance à la marginalisation}

On a vu dans les précédents paragraphes que la beauté "féminine " recherchée par la plupart des transgenres wallisiennes est une féminité de type "moderne et "cosmopolite». Ainsi, à l'ins- tar de leurs " consoeurs" polynésiennes (voir, par exemple, Besnier, 1997 et 2002, sur les leiti de Tonga et Elliston, 2014 à propos des raerae de Tahiti), la plupart des transgenres wallisiennes de Nouméa, et principalement les plus jeunes, cherchent à incarner un modèle de féminité cosmopolite à travers leurs styles de robe volontairement voyantes - des robes fashion qui couvrent à peine leurs parties intimes, leur imitation de l'allure des mannequins (un pied placé précisément devant l'autre lors de la marche), et un mode de vie consumériste et urbain (manger dehors, sortir faire la fête dans les lieux nocturnes les fins de semaine, etc.).

Par ailleurs, depuis les années 2000, nombre d'entre elles envisagent ou ont déjà effectué des opérations de transition chirurgicale (mammoplastie, vaginoplastie) afin d'avoir un corps «féminin " adhérant ainsi aux nouvelles techniques esthétiques et médicales modernes, rendues possible par l'expansion d'un tourisme international de chirurgie esthétique transgenre en Thaillande (Aizura, 2010 et 2011). Amira, à titre d'exemple, bénéficie ainsi d'une certaine popularité parmi les transgenres à Nouméa. Cheveux longs, grande taille, corps sexuellement transformé par une chirurgie de réassignation de sexe, maquillage soigné et habillement "attirant ", Amira affiche une féminité sophistiquée. Elle a aussi fait des études, ce qui n'est pas le cas de la plupart des transgenres ; cela lui permet d'exercer un emploi de "blancs ", et de gagner un "bon" salaire. Toutes ces caractéristiques font sa renommée. Elle incarne ainsi un modèle de réussite à suivre. Les plus jeunes transgenres sollicitent souvent son amitié en lui rendant visite ou en l'abordant dans le quartier ou en soirée. Les caractéristiques qui font l'admiration des «filles " envers Amira ont donc un rapport avec la réussite individuelle, le consumérisme, l'ascension sociale urbaine et ce que Besnier appelle "les aspirations à la modernité » (2013 : 154). Ces critères sont aussi recherchés et admirés par les autres membres de la diaspora wallisienne, hommes ou femmes, comme je l'ai souligné auparavant. Amira déclare en effet que l'attitude de ses parents et de ses soeurs a changé envers son " efféminement " après qu'elle eut fini ses études et qu' elle eut commencé à travailler, comme elle le souligne dans cet extrait :

"Quand j'ai commencé à travailler, là, c'est vraiment là, on va dire, [que] mes parents, ça y est, ils étaient fiers de moi. Et voilà ! Ça vient tout doucement [...]. »

Les « filles » transgenres stigmatisées réussissent ainsi à représenter une " réussite sociale " à travers leur travail et leur apparence soignée et sophistiquée, ce qui leur permet souvent de négocier leur acceptation. Il est ainsi fréquent d'observer chez les transgenres de Nouméa l'investissement de leur salaires, - ou de l'argent du tai'ata -, dans des biens et des modes de vie de consommation 
ostentatoires : téléphones, vêtements, chaussures, parfums, soins esthétiques, restaurants, sorties nocturnes le week-end, voyages, etc. Face à leur marginalisation sociale due à leurs performance de genre et de sexe, la manifestation d'un mode de vie "moderne" permet en effet aux transgenres wallisiennes, comme à leurs homologues polynésiennes (voir Besnier, 2002 et Elliston, 2014), de ne pas être exclues, dans une certaine mesure, des structures locales du statut et du "prestige " social. Elles expriment ainsi de plus en plus des désirs qui n'existent pas pour leurs aînés ou leurs homologues de Wallis-et-Futuna, ou que ces dernières considèrent hors de leur portée : désir de produits cosmétiques, de coiffure individualisée, et récemment, de se faire opérer chirurgicalement à l'étranger. Cependant, l'expression et la réalisation de ces désirs n'est rendue possible que par la vie urbaine où existent les centres de soin de corps, les boutiques qui vendent de la marchandise emportée, les différents lieux urbains de divertissement et de socialisation où peut se manifester cette féminité moderne. Cependant, il faudrait noter ici que seule une petite partie des transgenres peut accéder à ces marchandises et être visible dans ces lieux urbains : ce sont celles qui en détiennent les moyens financiers et qui le plus souvent appartiennent à la classe moyenne urbaine émergente.

\section{Conclusion}

La visibilité croissante, en Nouvelle-Calédonie, des transgenres d'origine wallisiennes - qui adoptent quotidiennement une apparence féminine - serait donc un fait récent qui a commencé à se manifester dans les années 1970. En effet, l'immigration polynésienne vers la Nouvelle-Calédonie, l'arrivée des travailleurs métropolitains, l'urbanisation et les changements socio-économiques sont à l'origine de la visibilité des transgenres dans les espaces publics, principalement dans les nouveaux lieux de divertissement qui ont commencé à voir le jour à cette période. Avec la modernisation de Nouméa, de nouveaux lieux de rencontre sont devenus disponibles dans la ville, alors qu'ils sont rares ou inexistants sur l'île d'origine de Wallis, comme les boîtes de nuit où la rencontre avec des hommes métropolitains peut avoir lieu, loin du contrôle familial ou de la " communauté ». En outre, l'arrivée de, et l'accès à des marchandises modernes telles que le rouge à lèvre, les talons aiguilles et les robes de soirée, ont permis le développement chez les transgenres de la performance d'une féminité d'influence occidentale, à travers les vêtements et les accessoires. Au cours des dernières décennies, Nouméa a connu de nouvelles mutations socio-économiques telles que l'expansion du salariat chez les hommes et les femmes, la généralisation d'un modèle urbain calqué sur un modèle global (sorties, vêtements à la mode, mixité, relations avant le mariage...). Ces mutations ont eu un impact, comme pour les autres membres de la société, sur la conception de la féminité et sur sa manifestation par les transgenres wallisiennes. Les transgenres de Nouméa furent ainsi les premières à adhérer aux symboles et aux modes de vie urbains de consommation d'influence occidentale pour manifester leur nouvelle féminité : vêtements à la mode, maquillage, goût pour la musique et les films occidentaux, visibilité collective dans les lieux urbaine (boîtes de nuit, bars, ...). Cette recherche de la "modernité " par les transgenres, à travers la performance de genre et le mode vie urbain, est souvent admirée par les membres de leur communauté, dans la mesure où elle représente une réussite sociale et l'augmentation du pouvoir d'achat, valeurs devenues importantes chez les Wallisiens vivant en ville. Cela permet à certaines d'entre elles de résister à la stigmatisation, même si leur féminité et leur mode de vie peuvent quelquefois être jugés "immoraux " et " irrespectueux ». Néanmoins, cette grande visibilité en ville ne doit pas nous faire oublier qu'elle n'est accessible qu'aux transgenres qui détiennent les ressources nécessaires pour y accéder. Les transgenres plus jeunes ou issues de familles précaires sont en marge de cette visibilité " collective " dans l'espace urbain. En affichant une féminité non soignée ou discrète, ces transgenres sont stigmatisées et ridiculisées par leur famille, en même temps que par les autres transgenres qui les jugent moins belles et non affirmées.

\section{BIBLIOGRAPHIE}

Académie Tahitienne-Fare Vāna’a, 1999. Dictionnaire Tahitien-Français (http://www.farevanaa.pf/ dictionnaire.php ; consulté en janvier 2013).

Aizura Aren Z, 2010. Feminine Transformations: Gender Reassignment Surgical Tourism in Thailand, Medical Anthropology: Cross-Cultural Studies in Health and Illness 28 (4), pp. 424-443 (http:// www.arenaizura.com/uploads/aizura_medical_ anthropology.pdf ; consulté en mars 2015).

-, 2011. The Romance of the Amazing Scalpel: 'Race', labour and affect in Thai gender reassignment clinics, in P. A. Jackson (ed.), Queer Bangkok, Hong Kong, Hong Kong University Press, pp. 142-162 (http://arenaizura. com/uploads/aizura_romance_scalpel.pdf ; consulté en mars 2015).

Alexeyeff Kalissa and Niko Besnier, 2014. Gender on the Edge: Identities, Politics, Transformations, in N. Besnier and K. Alexeyeff (eds), Gender on the Edge: Transgender, Gay, 
and Other Pacific Islanders, Honolulu, University of Hawai'i Press.

Angleviel Frédéric, 2006. Wallis-et-Futuna (19421961) ou comment le fait migratoire transforma le protectorat en том, Journal de la Société des Océanistes 122-123: Spécial Wallis-et-Futuna (I. Leblic et H. Guiot éds), pp. 61-76 (http: // jso.revues.org/541; consulté septembre 2014).

BAuer François, 2002. Raerae de Tahiti: Rencontre du troisième type, Papeete, Haere po.

Besnier Niko, 1994. Polynesian gender liminality, in G. Herdt (ed.), Third sex, third gender, New York, Zone, pp. 285, 328.

—, 1997. Sluts And superwomen: The politics of gender liminality in urban Tonga, Ethos 62 (1-2), pp. 5-31.

-, 2013. Modernité, corps et transformation de soi, Terrain 61, pp. 150-165 (http://terrain. revues.org/15229, consulté en août 2017).

Deckker Paul (de), 1997. Uvea-Wallis: Une identité mouvementée, in T. Darell et P. Deckker (éds), Identités en mutation dans le Pacifique à l'aube du troisième millénaire (Hommage à Joël Bonnemaison 1940-1997), Bordeaux, Centre de recherches sur les espaces tropicaux de l'Université Michel de Montaigne-Bordeaux 3, coll. Îles et archipels 26.

Dolgoy Reevan, 2000. The search for recognition and social movement emergence: Towards an understanding of the transformation of the fa'afafine of Samoa, PhD thesis, Department of Sociology, University of Alberta, Canada.

Dussy Dorothée, 1998. Nouméa et les squats. De la construction urbaine coloniale aux occupations océaniennes spontanées: vers le partage spatial et symbolique de la ville, thèse de doctorat d'anthropologie historique et sociale, École des hautes études en sciences sociales.

Dussy Dorothée et WitTersheim Éric (éds), 2013. Villes invisibles. Anthropologie urbaine $d u$ Pacifique, Paris, L'Harmattan, cahiers du Pacifique Sud contemporain.

Elliston Deborah, 2014. Queer History and its Discontents at Tahiti. The Contested Politics of Modernity and Sexual Subjectivity, in N. Besnier and K. Alexeyeff (eds), Gender on the Edge: Transgender, Gay and other Pacific Islanders, Honolulu, University of Hawai i Press, pp. 33-55.

Freyss Jean, 1995. Économie et changement social en Nouvelle-Calédonie, Paris, PUF, collection IEDES-Tiers-monde.
Guiart Jean, 1996. Nouméa, cité métisse ?, Journal de la Société des Océanistes 103 (2), pp. 231273 (http://www.persee.fr/doc/jso_0300-953x_ 1996_num_103_2_1992).

Hamelin Christine, 2000. Les gens de Nouméa. Mutations et permanences en milieu urbain, in A. Bensa et I. Leblic (éds), En pays kanak, Paris, Mission du Patrimoine ethnologique et Éditions de la Maison des sciences de l'homme, coll. Ethnologie de la France, cahier 14, pp. 339-354.

Kulick Don, 1998. Travesti: Sex, gender, and culture among Brazilian transgender prostitutes, Chicago, University of Chicago Press.

Kuwahara Makiko, 2014. Living as and living with Māhu and Raerae: Geopolitics, Sex, and Gender in the Society Islands, in N. Besnier and K. Alexeyeff (eds), Gender on the Edge: Transgender, Gay and other Pacific Islanders, Honolulu, University of Hawai'i Press, pp. 93114.

Leleivai Hapakuke Pierre, 2002. Communication, politique et trajectoires identitaires à Wallis-et-Futuna, Hermès 1 (32-33), pp. 181 189 (http://www.cairn.info/revuehermes-larevue - 2002-1-page-181.htm ; consulté en août 2013).

Marmouch Maroua, 2015. Transgenres en Nouvelle-Calédonie. Discussions intimes sur des parcours de vie wallisiens et quelques parcours kanak, thèse de doctorat, cotutelle École des hautes études en sciences sociales et Université de Turin.

Pechberty Dominique, 2004. Les Uvéens en Nouvelle-Calédonie, The Journal of Pacific Studies 1, pp. 63-81.

Roux Jean-Claude, 1985. Un exemple de migration-enracinement dans le Pacifique sud: la communauté wallisienne et futunienne de Nouvelle-Calédonie, in ORSTOM, Cahiers des Sciences humaines XXI (4), pp. 461-480.

Salomon Christine, 2000. Hommes et femmes. Harmonie d'ensemble ou antagonisme sourd ?, in A. Bensa et I. Leblic (éds), En pays kanak. Ethnologie, linguistique, histoire, archéologie en Nouvelle-Calédonie, Paris, Mission du Patrimoine ethnologique et Éditions de la Maison des sciences de l'homme, coll. Ethnologie de la France, cahier 14, pp. 311-338.

Schmidt Johanna Mary, 2005. Migrating genders westernisation, migration, and Samoan Fa'afafine, $\mathrm{PhD}$ thesis Sociology, The university of Auckland. 
Wilson Ara, 2011. Medical tourism in Thailand, in A. Ong and N. Chen (eds), Asian Biotech, Durham, NC, Duke University Press. 